\title{
The CHREST Architecture of Cognition The Role of Perception in General Intelligence
}

\author{
Fernand Gobet \\ Centre for the Study of Expertise \\ Brunel University \\ Uxbridge Middlesex, UB8 3PH \\ fernand.gobet@brunel.ac.uk
}

\author{
Peter C.R. Lane \\ School of Computer Science \\ University of Hertfordshire \\ Hatfield, Hertfordshire, AL10 9AB \\ peter.lane@bcs.org.uk
}

\begin{abstract}
This paper argues that the CHREST architecture of cognition can shed important light on developing artificial general intelligence. The key theme is that "cognition is perception." The description of the main components and mechanisms of the architecture is followed by a discussion of several domains where CHREST has already been successfully applied, such as the psychology of expert behaviour, the acquisition of language by children, and the learning of multiple representations in physics. The characteristics of CHREST that enable it to account for empirical data include: selforganisation, an emphasis on cognitive limitations, the presence of a perception-learning cycle, and the use of naturalistic data as input for learning. We argue that some of these characteristics can help shed light on the hard questions facing theorists developing artificial general intelligence, such as intuition, the acquisition and use of concepts, and the role of embodiment.
\end{abstract}

\section{Introduction}

There are two main broad approaches for developing general artificial intelligence. The first is to use whatever techniques are offered by computer science and artificial intelligence, including brute force, to create artefacts that behave in an intelligent way. The second is to develop computational architectures that closely simulate human behaviour in a variety of domains. Examples of this approach include ACTR (Anderson and Lebière, 1998), Soar (Newell, 1990), and EPAM (Feigenbaum and Simon, 1984). More recently, the computational architecture CHREST (Chunk Hierarchy and REtrieval Structures) (Gobet et al., 2001; Gobet and Simon, 2000; Lane, Cheng, and Gobet, 2000) has simulated data in a number of domains, including expert behaviour in board games, problem solving in physics, first language acquisition, and implicit learning.

The strength of cognitive architectures is that their implementation as computer programs ensures a high degree of precision, and offers a sufficiency proof that the mechanisms proposed can carry out the tasks under study something obviously desirable if artificial general intelligence is the goal. The extent to which success is reached in simulating actual human behaviour can be assessed by using measures such as eye movements, reaction times, and error patterns.

The aim of this paper is to introduce CHREST, to illustrate the kind of phenomena it has already been able to successfully simulate, and to show what insight it offers on the creation of AI systems displaying general intelligence. The claim made here is that developing a cognitive architecture - and thus understanding human intelligence provides critical insight for developing general artificial intelligence.

\section{The CHREST Architecture}

Just like its predecessor, EPAM (Elementary Memorizer and Perceiver) (Feigenbaum and Simon, 1984), CHREST assumes the presence of short-term memory (STM) and long-term memory (LTM) structures, and models cognition as the product of the interaction of perceptual learning, memory retrieval, and decision-making processes. A central theme is that "cognition is perception" (De Groot and Gobet, 1996). Thus, the architecture postulates a close interaction between perception, learning, and memory: CHREST's knowledge directs attention and perception, and, in turn, perception directs the learning of new knowledge.

Another essential aspect of the architecture is that Simon's assumption of bounded rationality (Simon, 1969) is taken very seriously. For example, CHREST's behaviour is constrained by the limited capacity of visual short-term memory (3 chunks), the relatively slow rate at which new elements can be learned ( 8 seconds to create a new chunk), and the time it takes to transfer information from LTM to STM (50 ms). Just like the human cognitive system, CHREST satisfices, and this might be a key condition for displaying general intelligence. All cognitive operations carried out by the system have a cost, which is indicated with approximate but fixed time parameters. The presence of these parameters enables close comparison between human and simulated behaviour (see De Groot and Gobet, 1996, for details; a technical specification of CHREST can be found at www.CHREST.info).

The emphasis on cognitive limitations is in stark contrast with architectures such as Soar, where the stress is on carrying out complex intelligent behaviour without imposing many constraints on the architecture (for example, Soar enjoys an unlimited capacity for its working memory). Compared to other architectures, CHREST might thus appear as a very austere system. However, it is also a powerful dynamic system governed not only by built-in capabilities 
but also, more importantly, by the complexities of its interaction with the environment. Together, these features enable it to cover a wide range of behaviours.

\section{Components}

The three main components of CHREST are shown in Figure 1: (a) mechanisms and structures for interacting with the external world; (b) multiple STMs that hold information from varied input modalities; and (c) an LTM, which holds information is in a "chunking network." A chunking network is a discrimination network whose dynamic growth is a joint function of the previous states of the system and the inputs from the environment. The visual input-output channels (and the STM associated with them) have been investigated in models of chess expertise where the actual eye movements of masters and novices have been simulated (De Groot and Gobet, 1996). In general, this simulated eye is crucial in understanding the interaction between low-level information, such as visual stimuli, and high-level cognition, such as concepts. The auditory channels have been investigated in a model of vocabulary acquisition (Jones, Gobet and Pine, 2007, 2008), which simulates the way children learn words using phonemic information.

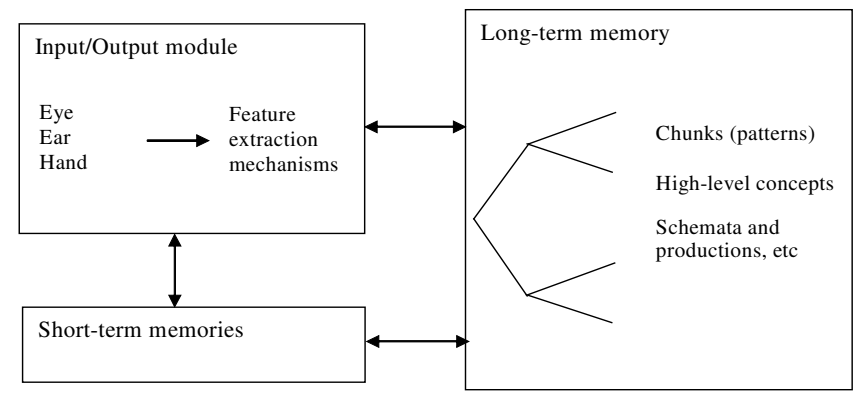

Figure 1. Key components of the CHREST architecture

\section{Learning Mechanisms}

Chunking networks are grown by mechanisms similar to those used in EPAM. A process called discrimination creates new nodes and a process called familiarisation incrementally adds information to existing nodes. An important extension, compared to EPAM, is a mechanism for creating high-level structures from perceptual information, by a process called template formation. The creation of a template, which is a kind of schema, uses both stable information (for creating its core) and variable information (for creating its slots). Templates are essential for explaining how chess Masters can recall briefly presented positions relatively well, even with a presentation time as short as 1 or 2 seconds (Gobet and Simon, 2000). They are also important for explaining how chess masters carry out planning - that is search at a level higher than that of moves. Another important novelty is the creation of lateral links (similarity links, production links, equivalence links, and generative links) between nodes (see Gobet et al., 2001, for detail). It is important to point out that all these mechanisms are carried out automatically. Much more than with previous chunking models (for example, Simon and Gilmartin, 1973), CHREST explains the development of expertise by both acquiring a large number of knowledge structures and building connections between them.

\section{Eye Movements and the Perception-Learning Cycle}

The frame problem is a central issue in cognitive science and artificial intelligence: How can a system notice the relevant changes in the environment in real time whilst ignoring the indefinite number of changes that are irrelevant? CHREST's solution consists of three parts, which all lead to a reduction in information. First, the limited capacity of the visual field eliminates a considerable amount of information coming from the environment. Second, the knowledge that the system brings to bear - and sometimes the lack of such knowledge - further constrains the amount of information processed. Third, the limited memory capacity we have mentioned earlier causes a further reduction of information. Thus, CHREST is highly selective, as presumably is the human cognitive system. This is consistent with research on perception and evolution, which has shown that animals and humans in particular have evolved powerful perceptual mechanisms for extracting key features from sensations in order to survive complex and dangerous environments.

Indeed, with CHREST, the link between perception and cognition is so tight that the distinction between these two sets of mechanisms all but disappears. To begin with, the focus of attention determines what information will be learned. Then, when possible, eye movements and thus attention will be directed by previous knowledge, making it more likely that the system pays attention to critical information. The key assumption here is that features that were important in the past - to the point that they led to learning - should be important in the future as well. This perception-learning-perception cycle is another way by which CHREST addresses the frame problem, as it leads to a selectivity of attention that further reduces the amount of information extracted from the environment and makes it possible to respond in real time.

\section{Some Domains Modelled by CHREST}

\section{Chess Expertise}

Historically, chess has been a standard domain for studying cognition, including intelligence, both for humans and computers. Chess was the first domain of application of CHREST, a domain that turned out to be excellent as it engages various cognitive abilities including perception, memory, decision making, and problem solving. It turns out that CHREST can simulate a large number of phenomena related to chess expertise. These include: the eye movements of novices and chess Masters (see Figure 2); recall performance in numerous memory experiments (including errors and the detail of the piece placements); and evolution 
of look-ahead search as a function of skill (De Groot and Gobet, 1996; Gobet, 1997; Gobet and Simon, 1996, 2000). The main mechanism explaining these phenomena is the acquisition of a large number of chunks (more than 300,000 for simulating Grandmasters) and templates, which are autonomously acquired from scanning master-level games.

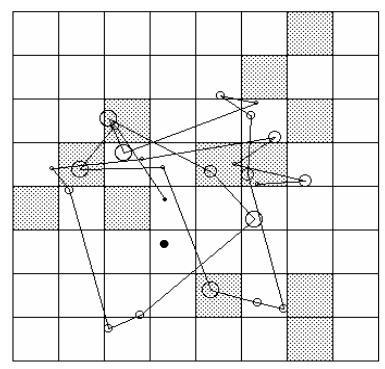

Human Master

$260 \mathrm{msec}$

$100 \mathrm{msec}$

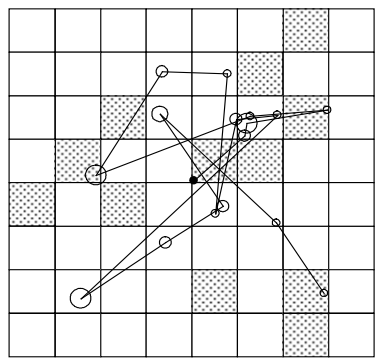

CHREST Master

$272 \mathrm{msec}$

$97 \mathrm{msec}$
Figure 2. Example of a Master's eye movements (left) and their simulations by CHREST (right). The mean and the standard deviation of fixation times are across all positions and all master subjects. (After De Groot and Gobet, 1996.)

Computational modelling is often criticised by describing it as only some kind of curve-fitting exercise, without new predictions and thus without real understanding. CHREST does not suffer from this weakness, as it has made a number of new predictions, some of which have been later confirmed by empirical data. A good example of this is the recall of random chess positions, which are constructed by haphazardly replacing the pieces of a game position on the board. When these simulations were carried out, the universal belief, based on research by Chase and Simon (1973) with a small sample, was that there was no skill difference in the recall of random positions, while of course Masters vastly outperform weaker players with game positions. The simulations repeatedly showed that there should also be a skill effect with this type of positions - for the simple reason that CHREST could recognize some chunks in random positions, just by chance, and that this was more likely with large networks of chunks. This prediction was verified by a re-analysis of all studies carried out with this type of material and by collecting new data (Gobet and Simon, 1996). Figure 3 shows the predictions of CHREST and the human data, for both game and random positions. (Please note the close fit between CHREST and the human data.) CHREST's predictions were also supported when random positions were created using different procedures (Gobet and Waters, 2003).

These results indicate that Masters perceive patterns in spite of the fact that the positions do not contain much structure, a further indication, if necessary, that chunking mechanisms are automatic and implicit. If this is the case, CHREST should be able to simulate the kind of phenomena observed in the implicit learning literature (Reber, 1967). In an implicit-learning experiment, stimuli generated from an artificial grammar are first shown to the subjects, and then subjects have to classify new strings as well-formed or not. The debate in this literature is whether subjects learn anything in these experiments, and, if so, what kind of things are learnt: rules, fragments, or exemplars? Unpublished work with Daniel Freudenthal indicates that CHREST can simulate some of the key results very well, suggesting that subjects in these experiments learn implicitly and unconsciously small fragments of stimuli that become incrementally larger with additional learning.

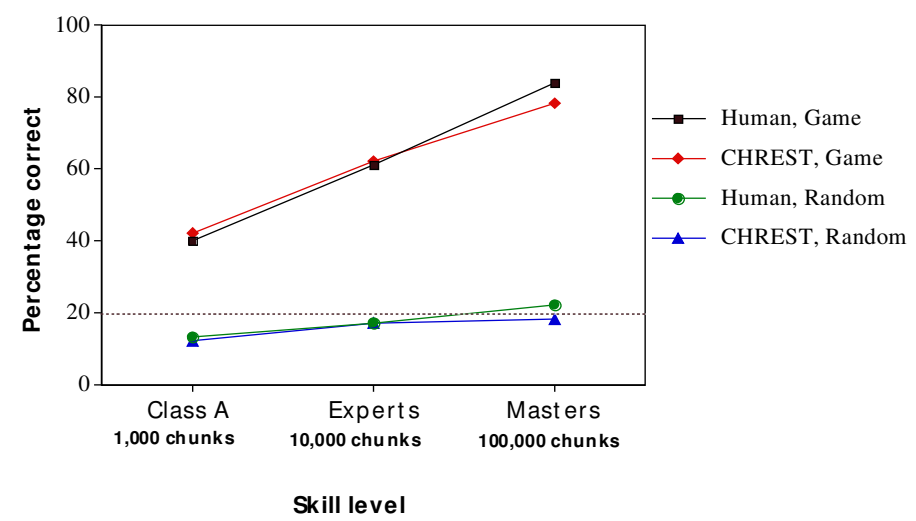

Figure 3. Memory for game and random positions as a function of skill level. The human data are the average results aggregated across 13 studies. (After Gobet and Simon, 1996.)

Of course, the essence of chess skill is not to memorize chess positions, but to find good moves. Work has also been done with CHREST to understand how human masters are able to find good moves despite searching only one very small subset of the search space. A first program inspired by CHREST, CHUMP (CHUnking of Moves and Patterns; Gobet and Jansen, 1994), was able to learn an association of chess moves to perceptual patterns. It is interesting that CHUMP, while playing at a fairly low level, performed better in positions requiring a 'positional judgement' than in tactical positions, which engage more look-ahead search. As positional judgment in chess is seen as a clear-cut example of intuition (e.g., Dreyfus \& Dreyfus, 1986), it could be argued that CHUMP captures this aspect of human intelligence (see also Simon, 1979). A second program, SEARCH (Gobet, 1997), simulated key parameters of the way human of different levels search a position. These parameters included depth of search, width of search, and rate at which moves were generated. More recent work with CHREST has added mechanisms combining look-ahead search and pattern recognition, paving the way to complete simulation of expertise in chess.

\section{Other Domains of Expertise}

An application of CHREST to the African game of Awele (Gobet, 2009) shows that it can play at a fairly good level by 
pattern recognition only, while at the same time simulating several results from memory-recall experiments. Simulations have also been carried out on memory for computer programs and the acquisition of multiple representations in physics. In this latter work, the aim was to study the acquisition of multiple diagrammatic representations, and the combination of these multiple representations to form problem-solving stages. The representations were, first, the classic circuit-style of representation, found in textbooks, and second, a specialized problem-solving representation, containing quantitative properties of the domain (see Lane et al., 2000 for details). The essential elements of the model here were the movements of the eye and visual short-term memory; some chunks were learnt for each representation, and they were combined within short-term memory using lateral links. These links are used in solving new problems, to retrieve known components of a problem.

Although simple in comparison to the abilities of ACT-R or Soar, this work provides CHREST with a rather unique form of problem solving, based around perceptual chunks. The idea is that problem solutions are planned, at a high level, by retrieving familiar chunks; these familiar chunks have been acquired by solving smaller problems in isolation. The solution process involves a form of composition, guided by the perceived separation of the problem into chunks. The decomposition used by CHREST corresponds with that used and drawn by human participants, providing empirical support for the role of perceptual chunks in problem solving.

\section{Linking Perception to Expectations}

Perception in CHREST is seen as a cycle, with the eye guided to look at parts of the scene or image where useful information is expected to lie. With human, and perhaps animal, cognition, it is expected that such expectations would be formed from information in more than one modality. For example, knowing a sequence of verbal statements may help guide the location of a sequence of perceptual icons.

Lane, Sykes and Gobet (2003) explored this aspect of cognition by training CHREST to encode information in more than one modality: visual and verbal. The process is illustrated by Figure 4. Here, one visual and one verbal stimulus are separately experienced and sorted through the long-term memory. Pointers to the retrieved chunks are placed into short-term memory. Because short-term memory is separated for each modality, the information about visual and verbal chunks is kept distinct. An association is then made between the visual and verbal memories.

The interaction between the two memories produces various measurable effects. For instance, prior expectations can improve the speed and accuracy with which unclear stimuli are recognised. The ultimate aim is to understand how low-level and high-level knowledge interact to produce intelligent behaviour, a question that has nagged cognitive science for decades (for example, Neisser, 1966).

Current work is looking to apply chunking mechanisms to bitmap-level data, whilst maintaining interactions between verbal and visuo-spatial information. One technique for doing so lies in employing techniques from component-based vision, where one layer of feature detectors seeks out symbolic features to pass to a second layer. For example, Han et al. (2009) employ support-vector machines to locate components of a human face, which are then classified by a separate classifier. A natural extension is to use CHREST as the second layer, and so gain the benefits both of low-level pixel analysis and high-level symbolic pattern matching across multiple representations.

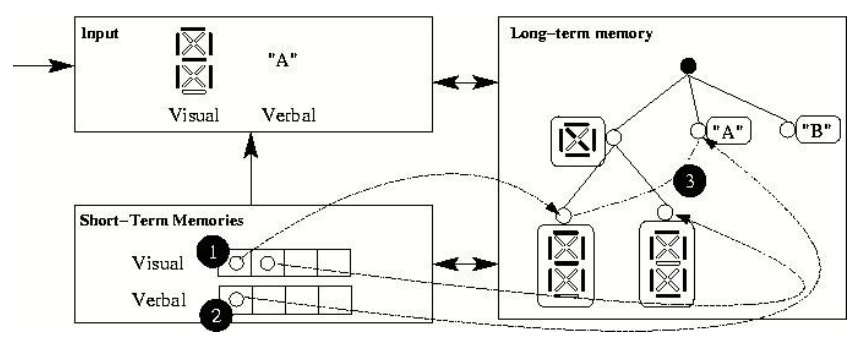

Figure 4. Learning to link information across two modalities. (1) The visual pattern is sorted through LTM, and a pointer to the node retrieved placed into visual STM. (2) The verbal pattern is sorted through LTM, and a pointer to the node retrieved placed into verbal STM. (3) A naming link is formed between the two nodes at the top of the STMs. (After Lane, Sykes and Gobet, 2003.)

\section{Acquisition of Language}

Language is uniquely human, and understanding how children acquire their first language will tell us something important about intelligence in general. We see language acquisition as one type of expertise, and argue that children become experts in their native language through implicitly acquiring a large number of chunks and links between them. A first variation of CHREST has studied the acquisition of vocabulary (Jones et al., 2007). The interest has been on how mechanisms in short-term memory and long-term memory interact through the creation and use of chunks. A second variation of CHREST, known as MOSAIC (Model of Syntax Acquisition In Children), has simulated with great success a number of empirical phenomena in the early acquisition of syntactic categories (Freudenthal et al., 2008, 2009). Our attention has focused on the "optional infinitive" phenomenon and related phenomena, such as the misuse of pronouns. The "optional infinitive" phenomenon concerns typical errors made by children in their use of finite (for example, goes, went) and non-finite verb forms (for example, go, going). For example, a child would say "her do it" instead of "she does it." A combination of four features makes the MOSAIC project unique within cognitive science: (a) it uses naturalistic input (utterances spoken by parents interacting with their children in a play setting); (b) it can simulate in detail the pattern and developmental trend of errors; (c) it uses exactly the same model for reproducing a number of empirical phenomena; and (d) it carries out simulations in several languages (so far, English, Dutch, German, French, Spanish; and Q'anjobalan, a Mayan language) with exactly the same model - the only difference 
being the maternal input used for training. Compared to other approaches in the field (e.g., connectionism), the extent of MOSAIC's coverage is striking, in particular when one considers that only simple and local learning mechanisms are used. In a nutshell, three interacting factors are essential for explaining MOSAIC's behaviour: rote learning, the creation and use of generative links, and the statistical structure of the input.

\section{General Intelligence: Some Central Questions}

Although CHREST does not yet show fully general intelligence, it offers a cognitive architecture whose combined components have proved sufficient to exhibit behaviour that is, in several domains, remarkably close to human behaviour. In this final section, we explore some central questions in artificial intelligence and cognitive science for which, we believe, CHREST provides important insight.

\section{The Role of Concepts}

It is generally accepted that acquiring and using concepts is essential for the survival of organisms and their successful interaction with the environment. Without concepts, organisms can show only primitive behaviour, as the lack of generalization means that each new exemplar must be treated separately. A considerable amount of experimental research has been carried out on concept formation and categorization in psychology over the last decades. We know a great deal about how people acquire and utilise concepts, with a number of computational models accounting for different aspects of these empirical data. For example, Gobet et al. (1997) used a model close to CHREST to investigate the role of strategies in concept formation, and the suitability of CHREST for simulating categorization experiments was further established by Lane and Gobet (2005).

The previous simulations with CHREST have essentially relied on individual nodes to account for the concepts used by humans. However, the type of chunking networks created by CHREST suggests other possibilities as well. A natural interpretation of these networks is that concepts do not map into single chunks (or even single templates), but rather correspond to a subset of nodes interlinked, to varying degrees, by lateral links. In this view, concepts are much more distributed than in standard symbolic models, and take on some of the flavour of how concepts are represented in connectionist networks; this idea is expanded upon in Lane, Gobet and Cheng (2000). While this idea is not new, the advantage of using CHREST is to provide mechanisms explaining how nodes and the links between them are acquired autonomously and in real time, how they relate to perceptual input, how they are influenced by the structure of the environment, and how they may integrate information across different modalities.

\section{Embodiment}

Although the first experiments with autonomous robots are fairly old, going back to Grey Walter's (1953) seminal work, it is only in the last twenty years or so that the field of embodied cognition has been taken up. A number of mobile robots have been created that are able to carry out fairly simple tasks (Pfeifer and Scheier, 1999). However, as we have argued elsewhere (Lane and Gobet, 2001), a limitation of current research in this field is that it does not provide mechanisms for how simple behaviours can be linked with more complex behaviours. The limitation is serious, as it is obvious that systems showing general intelligence must have (at least) these two levels of complexity. Specifically, the lack of symbolic processing in current embodied-cognition systems means that there are important limits in the kind of behaviours that can be addressed.

As soon as one tries to link simple behaviours with symbolic processes, the question of symbol grounding arises. The CHREST framework provides a fairly simple explanation for this: as noted above, symbols, that is chunks, are grounded through perception. Importantly, as mentioned above, this idea goes much beyond that of simply linking symbols to external objects, through perception. Chunks also shape how the world is perceived, in two important ways. First, they determine how percepts will be organized. The way former world chess champion Gary Kasparov perceptually groups information of a chess position is different from the way an amateur does. Second, chunks actively direct eye movements and thus determine to which part of the display attention will be heeded.

To show how such an integrated system would work, we are currently working to implement CHREST into a mobile robot. Our first aim is to show that a chunking-based architecture can replicate some of the 'classic' simulations in the literature, such as the avoidance of obstacles and the emergence of complex behaviour. Our belief is that combining symbolic and non-symbolic approaches within an embodied system is likely to have important consequences, both for theory and application. In particular, symbolic information will enable the robot to be 'articulate', explaining, verbally, what and why it takes particular decisions.

\section{Conclusion}

We have argued that progress towards understanding general intelligence requires an integrated approach: not just integrating perception with learning, but also integrating high-level and low-level modes of processing. It is unlikely that a satisfactory theory of human intelligence will be developed from a single explanatory framework, so we expect an artificial system with claims to general intelligence to be a unification of diverse approaches.

In this paper, we have set out the case for a symbolic system, which integrates perception with learning. We have shown how the system captures many details of high-level processing in human cognition, and also how it captures physical behaviours, such as details of eye fixations. These successes allow us to propose some ideas of how a complete model of the mind may look. 
Central would be a two-way interaction between perception and cognition. This interaction must be coupled with an incremental learning system, capable of acquiring a vast and coherent structure of nodes and links. But also, paradoxically perhaps, the architecture should exhibit strong constraints, such as limited processing time or short-term memory capacities. These limits lie behind some of the key empirical challenges to computational theories of psychological behaviour.

\section{Acknowledgements}

This research was supported by the Economics and Social Research Council under grant number RES-000-23-1601.

\section{References}

Anderson, J. R., and Lebière, C. (Eds.). (1998). The atomic components of thought. Mahwah, NJ: Erlbaum.

Chase, W. G., and Simon, H. A. (1973). Perception in chess. Cognitive Psychology, 4, 55-81.

De Groot, A. D., and Gobet, F. (1996). Perception and memory in chess: Heuristics of the professional eye. Assen: Van Gorcum.

Dreyfus, H., and Dreyfus, S. (1986). Mind over machine. New York: Free Press.

Feigenbaum, E. A., and Simon, H. A. (1984). EPAM-like models of recognition and learning. Cognitive Science, 8, 305-336.

Freudenthal, D., Pine, J. M., Aguado-Orea, J. \& Gobet, F. (2007). Modelling the developmental patterning of finiteness marking in English, Dutch, German and Spanish using MOSAIC. Cognitive Science, 31, 311-341.

Freudenthal, D., Pine, J. M., \& Gobet, F. (2009). Simulating the referential properties of Dutch, German and English Root Infinitives in MOSAIC. Language Learning and Development, 5, 1-29.

Gobet, F. (1997). A pattern-recognition theory of search in expert problem solving. Thinking and Reasoning, 3, 291-313.

Gobet, F. (2009). Using a cognitive architecture for addressing the question of cognitive universals in crosscultural psychology: The example of awalé. Journal of Cross-Cultural Psychology, 40, 627-648.

Gobet, F., and Jansen, P. (1994). Towards a chess program based on a model of human memory. In H. J. van den Herik, I. S. Herschberg and J. E. Uiterwijk (Eds.), Advances in Computer Chess 7 (pp. 35-60). Maastricht: University of Limburg Press.

Gobet, F., Lane, P. C. R., Croker, S., Cheng, P. C.-H., Jones, G., Oliver, I., and Pine, J. M. (2001). Chunking mechanisms in human learning. Trends in Cognitive Sciences, 5, 236-243.

Gobet, F., Richman, H., Staszewski, J., and Simon, H. A. (1997). Goals, representations, and strategies in a concept attainment task: The EPAM model. The Psychology of Learning and Motivation, 37, 265-290.

Gobet, F., and Simon, H. A. (1996). Recall of rapidly presented random chess positions is a function of skill. Psychonomic Bulletin and Review, 3, 159-163.

Gobet, F., and Simon, H. A. (2000). Five seconds or sixty? Presentation time in expert memory. Cognitive Science, 24, 651-682.

Gobet, F., and Waters, A. J. (2003). The role of constraints in expert memory. Journal of Experimental Psychology: Learning, Memory and Cognition, 29, 1082-1094.

Grey Walter, W. The living brain (1953). London, UK: Penguin.

Han, J.W., Lane, P.C.R., Davey, N. and Sun Y. (2009). Attention mechanisms and component-based face detection. Proceedings of the International Conference on Methods and Models in Computer Science (IEEE Computer Society).

Jones, G., Gobet, F., \& Pine, J. M. (2007). Linking working memory and long-term memory: A computational model of the learning of new words. Developmental Science, $10,853-873$.

Jones, G., Gobet, F., \& Pine, J. M. (2008). Computer simulations of developmental change: The contributions of working memory capacity and long-term knowledge. Cognitive Science,32, 1148-1176.

Lane, P. C. R., Cheng, P. C.-H., and Gobet, F. (2000). CHREST+: Investigating how humans learn to solve problems using diagrams. AISB Quarterly, 103, 24-30.

Lane, P. C. R., and Gobet, F. (2001). Simple environments fail as illustrations of intelligence: A review of R. Pfeifer and C. Scheier: 'Understanding Intelligence'. Artificial Intelligence, 127, 261-267.

Lane, P. C. R., \& Gobet, F. (2005). Discovering predictive variables when evolving cognitive models. Third International Conference on Advances in Pattern Recognition.

Lane, P.C.R., Gobet, F. \& Cheng, P.C-H. (2000). Learningbased constraints on schemata. Proceedings of the TwentySecond Annual Conference of the Cognitive Science Society, pp. 776-81.

Lane, P. C. R., Sykes, A. K., and Gobet, F. (2003). Combining low-level perception with expectations in CHREST. In F. Schmalhofer, R. M. Young and G. Katz (Eds.), Proceedings of EuroCogSci 03: The European Cognitive Science Conference 2003 (pp. 205-210). Mahwah, NJ: Erlbaum.

Newell, A. (1990). Unified theories of cognition. Cambridge, MA: Harvard University Press.

Pfeifer, R., and Scheier, C. (1999). Understanding intelligence. Cambridge: MIT Press.

Reber, A. S. (1967). Implicit learning of artificial grammars. Journal of Verbal Learning and Verbal Behaviour, 6, 855-863.

Simon, H. A. (1969). The sciences of the artificial. Cambridge, MA: MIT Press.

Simon, H. A. (1979). Models of thought (Vol. 1). New Haven, CT: Yale University Press.

Simon, H. A., and Gilmartin, K. J. (1973). A simulation of memory for chess positions. Cognitive Psychology, 5, 29-46. 